\title{
A Novel FBG-Based Self-Seeded RSOA Transmitter with Noise Mitigation for Dense SS-WDM PONs
}

\author{
Ziad A. El-Sahn ${ }^{1,2}$, Walid Mathlouthi ${ }^{1}$, Habib Fathallah ${ }^{1,3}$, Sophie LaRochelle ${ }^{1}$, Leslie A. Rusch ${ }^{1}$ \\ ${ }^{1}$ COPL, ECE Dept., Université Laval, Québec, QC G1K 0A6, Canada, ziad elsahn@ieee.org \\ ${ }^{2}$ Photonics Systems Group, ECE Dept., McGill University, Montreal, QC H3A 2A7, Canada. \\ ${ }^{3}$ College of Engineering, ECE Dept., King Saud University, Riyadh, 11421, Saudi Arabia.
}

\begin{abstract}
We demonstrate dense SS-WDM uplink over legacy PONs using a novel self-seeded RSOA transmitter and a balanced receiver that preserves noise cleaning. 32 colorless ONUs can be supported at $1.25 \mathrm{~Gb} / \mathrm{s}$ without DCF and FEC.
\end{abstract}

\section{INTRODUCTION}

Reduced inventory cost is often advocated to support colorless wavelength-division multiplexed (WDM) passive optical network (PON) architectures using reflective semiconductor optical amplifier (RSOA)-based optical network units (ONUs) [1], [2]. Self-seeding have been proposed to boost the in-band power and to eliminate the need for centralized sources [3], [4]. However, when using incoherent sources for spectrumsliced (SS)-WDM, intensity noise limits the performance and incurs bit error rate (BER) floors, yielding a tradeoff between the spectral efficiency and the bit rate. A rich body of research has examined the use of the nonlinear dynamics of semiconductor optical amplifiers (SOAs) to mitigate the intensity noise of a separate thermal source [5], [6].

In this paper, for the first time, we use the RSOA in a combined role of thermal source and noise mitigation device. We confirm that a self-seeded RSOA signal also exhibits a significant reduction in intensity noise vis-à-vis a passively sliced thermal source. A partially reflective ( $p \%)$ fiber Bragg grating (FBG) is used for self-seeding in contrast with previous solutions. The reflectivity is optimized to tradeoff output power versus noise cleaning, to minimize the BER. For colorless ONU operation, the RSOA is remotely self-seeded by placing the FBG at the remote node $(\mathrm{RN})$. We use a recently proposed balanced receiver (BR) that preserves noise cleaning [6]. Our RSOA/BR combination offers tight dense WDM wavelength packing, and is therefore best targeted as an innovative method for gradually upgrading existing PONs to SS-WDM with no modification to the passive splitter RN.

\section{PON ARCHITECTURAL IMPACTS AND NOISE MitigATION THROUGH OUR RSOA/BR TRANSMITTER/RECEIVER PAIR}

Fig. 1(a) shows our proposed self-seeded RSOA transmitter incorporating a $p \%$ reflective FBG. The RSOA is self-seeded with a filtered version of its amplified spontaneous emission (ASE), while directly modulating its current. Re-injecting the RSOA with a certain waveband favors the output/gain of the RSOA into that band, lowering the out-of-band ASE and amplifying the desired channel as in Fig. 2(b). Clearly, a high FBG reflectivity guarantees that the RSOA is deeply saturated,

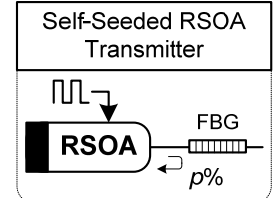

(a)

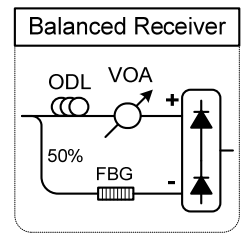

(b)
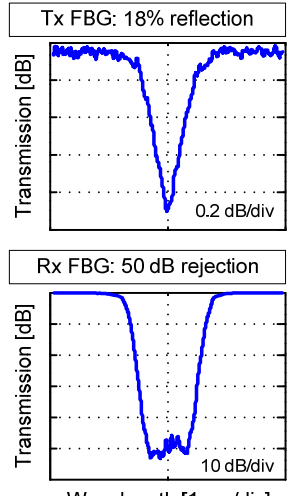

Wavelength [1 $\mathrm{nm} / \mathrm{div}]$
Fig. 1: (a) Proposed self-seeded RSOA transmitter and the self-seeding FBG response, (b) the balanced receiver with the notched filter FBG response (Rx: receiver, Tx: transmitter)

thus improving the noise suppression, but decreasing the output power. Whereas, a lower reflectivity increases the output power, but reduces the noise suppression. Therefore, we optimize the FBG reflectivity to balance these effects and minimize the BER. Recall that in previous studies no attempt was made to examine the noise mitigation [4].

In order for noise cleaning to carry through to the reception, optical filtering should be avoided [5]. In our proposal the self-seeding FBG is less than $20 \%$ reflective, therefore it is not a classical filter and the dynamics are much more complex. The filter is building up the output using feedback, working with the RSOA dynamics to achieve (not destroy) noise cleaning. Passive post filtering after our FBG does destroy this self-seeded noise cleaning as it does in other systems using SOAs for intensity noise cleaning.

At the receiver we use a recently proposed BR that preserves much of the noise cleaning [6]. The receiver structure is illustrated in Fig. 1(b). The upper arm is an all-pass filter and the lower arm is a notched filter FBG centered at the desired channel. A variable optical attenuator (VOA) and an optical delay line (ODL) are used for balancing. The transmission responses of the self-seeding FBG $(p=18 \%)$ and the notched FBG ( $50 \mathrm{~dB}$ rejection) are also shown in Fig. 1.

Previous demonstrations of this BR used separate broadband sources, SOAs for noise cleaning, and external modulators at the transmitter. Our proposal is the first examination and optimization, to our knowledge, of noise cleaning on a 


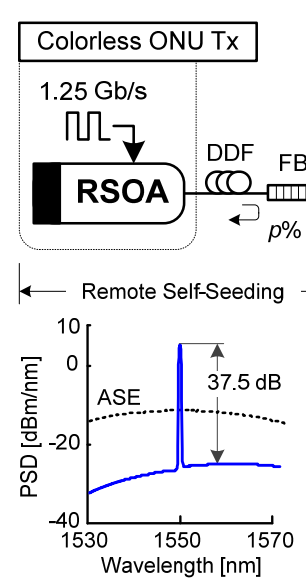

(b)
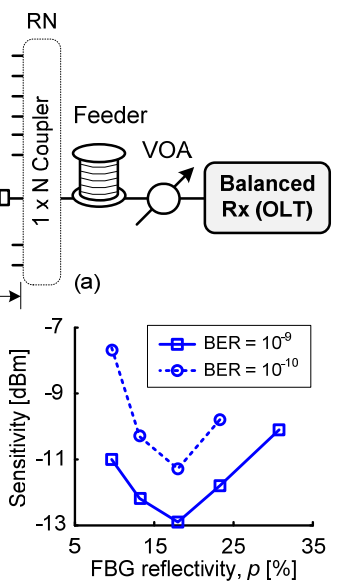

(c)
Fig. 2: (a) Experimental setup, (b) transmitter output PSD, (c) sensitivity versus reflectivity (ASE: amplified spontaneous emission, DDF: distribution drop fiber, OLT: optical line terminal, RN: remote node).

self-seeded RSOA. It greatly reduces the complexity and cost of previous architectures [6] as the RSOA performs modulation, amplification and noise cleaning simultaneously.

Our RSOA/BR combination has an interesting impact on the PON architecture. In traditional arrayed waveguide grating (AWG)-based WDM PONs, the passive filtering at the RN would negate noise cleaning advantages, unless extremely wide filtering is used (spectrally inefficient). Our approach uses the legacy passive splitter-based, single-feeder PON infrastructure (see the experimental setup in Fig. 2) allowing higher bit rate SS-WDM users to coexist with legacy users.

\section{Results, Discussions AND CONCLUSIONS}

The experimental setup used to demonstrate the uplink of our SS-WDM PON is shown in Fig. 2(a). Given the previously demonstrated robustness of BR to cross-talk [6], and the lack of equipment for multi-channel operation, we focus our attention on the single channel experimental validation. Our contribution is mainly finding the optimum balance of output power versus noise mitigation via the $p \%$ factor. At the ONU side; the RSOA is directly modulated at $1.25 \mathrm{~Gb} / \mathrm{s}$. A $2 \mathrm{~km}$ distribution drop fiber (DDF) separates the furthest ONU from its self-seeding FBG. We also examine the case with $0 \mathrm{~km}$ DDF to consider the nearest ONU. Standard single mode fiber (SMF-28) feeder lengths of 10 and $20 \mathrm{~km}$ are considered with no dispersion compensation. Since the RN contributes only to splitting losses, we forgo the $1 \times N$ coupler for the experiment. At the receiver a VOA is used to control the received power for the BER measurements. A JDS H301-2310 RF amplifier with $11 \mathrm{~dB}$ noise figure (no low noise amplifier was available in the laboratory), and a $4^{\text {th }}$ order $933 \mathrm{MHz}$ Bessel-Thomson low-pass filter are used after the balanced receiver.

The output power spectral density (PSD) of the transmitter with an $18 \%$ reflective FBG is shown in Fig. 2(b). The output power is $4.5 \mathrm{dBm}$ and $4 \mathrm{dBm}$ for the case of $0 \mathrm{~km}$ and $2 \mathrm{~km}$ DDFs, respectively, and the channel bandwidth is $25 \mathrm{GHz}$.

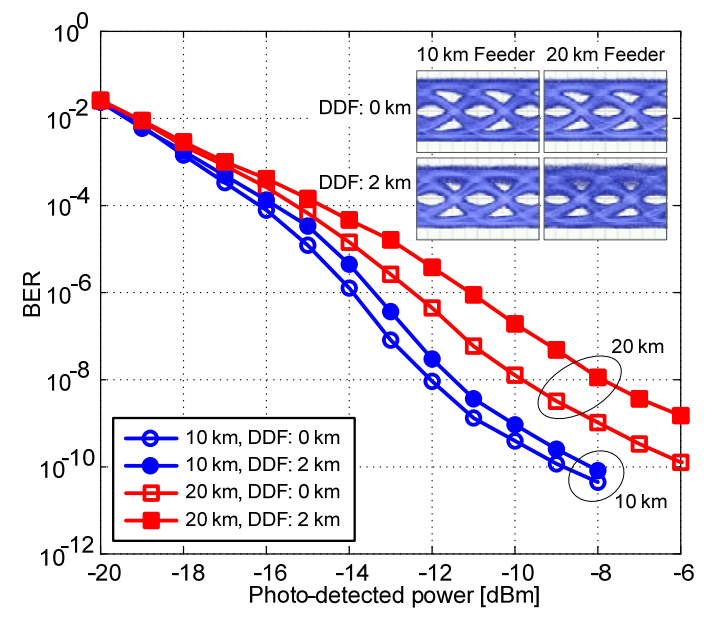

Fig. 3: BER performance at $1.25 \mathrm{~Gb} / \mathrm{s}$ per user.

The results of the FBG optimization are shown in Fig. 2 in terms of the sensitivity versus reflectivity, for $10^{-10}$ and $10^{-9}$ back-to-back BER. These results are valid for both the nearest and furthest ONUs. In our experiment we tested reflectivity values from $10 \%$ to $35 \%$, and found the optimum to be $18 \pm 2 \%$. This optimum is not unique; it depends on the characteristics of the RSOA and the channel $3 \mathrm{~dB}$ bandwidth. The key idea is to find the seed power that optimally balances self-seeding and noise cleaning.

The BER overall system performance using the optimum FBG is shown in Fig. 3 for $10 \mathrm{~km}$ and $20 \mathrm{~km}$ feeder lengths and for $0 \mathrm{~km}$ and $2 \mathrm{~km}$ DDF lengths. BER below $10^{-9}$ is achieved for both feeder lengths (eye diagrams at $-12 \mathrm{dBm}$ are shown as insets). We note a $3 \mathrm{~dB}$ power penalty from the 10 to the $20 \mathrm{~km}$ feeder which is mainly due to the additional dispersion. For the $10 \mathrm{~km}$ case, a $1 \mathrm{~dB}$ power penalty between the nearest and the furthest ONUs is measured, and a $2 \mathrm{~dB}$ penalty is imposed by the $20 \mathrm{~km}$ feeder. As the length of the DDF is increased, additional dispersion degrades the performance as seen from the eye diagrams.

While cross-talk is negligible in this study, a multi-channel system invokes significant splitting losses. Based on our results, we calculate the capacity of a multi-user system by generating a realistic power budget analysis when replacing our low performance JDS amplifier with a MITEQ AM-1300 low noise amplifier with only $1.6 \mathrm{~dB}$ noise figure. Clearly, the noise figure of an RF amplifier linearly affects the sensitivity; therefore the sensitivity is enhanced by $9.4 \mathrm{~dB}$ relative to our measurements. Up to 32 users can be supported over a $10 \mathrm{~km}$ feeder without a forward error correcting (FEC) code, and up to 16 users for a $20 \mathrm{~km}$ link.

\section{REFERENCES}

[1] F. Payoux et al., Proc. OFC'07, TuG5 (2007).

[2] J. H. Lee et al., J. Lightwave Technol. 28, 344 (2010).

[3] E. Wong et al., J. Lightwave Technol. 25, 67 (2007).

[4] N. Nadarajah et al., Photon. Technol. Lett. 19, 1559 (2007).

[5] A. D. McCoy et al., J. Lightwave Technol. 23, 2399 (2005).

[6] W. Mathlouthi et al., J. Lightwave Technol. 27, 5045 (2009). 